\title{
The semi-Euclidean approach in statistical mechanics. I. Basic expansion steps and estimates ${ }^{\star}$
}

\author{
Paul Federbush \\ Department of Mathematics, University of Michigan, Ann Arbor, Michigan 48104 \\ (Received 29 April 1975) \\ The semi-Euclidean formulation, developed in constructive quantum field theory to handle boson-fermion \\ models, is adapted to the statistical mechanics setting
}

\section{INTRODUCTION}

In Ref. $1 \mathrm{D}$. Brydges and I presented a formulation for treating boson-fermion models, taking powerful techniques for using Euclidean fields to study Boson models in constructive quantum field theory, and combining these with operator methods to handle the fermions. This, the semi-Euclidean approach, now seems like a useful framework to study such theories. In Ref. 2 Brydges studies the generalized Yukawa model using semi-Euclidean methods. In Ref. 3 techniques patterned after those in Refs. 1 and 2 were applied to prove the classic theorem of Dyson and Lenard ${ }^{4}$ on the stability of matter. In the present series of papers I intend to introduce a full semi-Euclidean formalism into statistical mechanics, furthering the flow of ideas from constructive quantum field theory into more classical fields of physics.

Ginibre has made beautiful applications of functional integration techniques in statistical mechanics. ${ }^{5}$ The formalism to be presented here will have many points in common with that of Ginibre. The interlacing we will see between the viewpoints of Refs. 1 and 5 seems very satisfying. The interactions in the stability of matter problem- $-1 / r$ forces between positively and negatively charged particles-would not lead to stability in the absence of the kinetic energy generated by the exclusion principle for the negative charges. ${ }^{6}$ This effect may be difficult to make explicit in the formalism of Ginibre, and the potentials considered in Ref. 5 exclude such forces. Our first long range goal will be to obtain the existence of the infinite volume correlation functions for the matter problem with the interaction $1 / r$ modified to $\exp (-n r) / r$ (this interaction still is excluded in Ref. $5)$. The extension to the long range $1 / r$ interaction is deferred.

Our avenue to the infinite volume correlation functions will hopefully be the adaptation of the cluster expansion of Glimm, Jaffe, and Spencer ${ }^{7}$ to the present formalism. In the present paper the cluster expansion is not developed. However, we do present some of the expansion operations to be used-differentiation of the exponent and a pull-through formula. The pull-through formula is used to generate a Ginibre-like expansion for correlation functions. We also present operator estimates substituting for the "defermiation" estimates of Ref. 1. These estimates - to be used in the proof of convergence of the cluster expansion-convert operator expressions to a context with commutivity. As an example we estimate some terms in the expansion of a correlation function.
The field theory of boson models, the field theory of boson-fermion models, and the field theory of second quantized matter in statistical mechanics provide three types of field theories-with striking similarities and striking differences - that will develop with mutual enrichment.

\section{NOTATION}

We work with $H$ of the form

$$
H=H_{0}+\frac{1}{2} \int d z_{1} d z_{2}: \bar{\psi} \psi\left(z_{1}\right) V\left(z_{1}, z_{2}\right) \bar{\psi} \psi\left(z_{2}\right):
$$

with $V$ symmetric in $z_{1}$ and $z_{2}$ and $H_{0}$ the sum of a multiple of the number operator with the kinetic energy form for Dirichlet data on the boundary of a fixed volume $V$. $\psi$ and $\bar{\psi}$ are annihilation and creation fields for a fermion or boson particle. The extension to more general potential interactions and more than one species of particle is straightforward (such as for protons and electrons moving in a fixed background charge). The objects from statistical mechanics we will study are of the form

$$
\operatorname{Tr}\left[\exp (-\beta H) \bar{\psi}\left(x_{1}, l_{1}\right) \cdots \psi\left(x_{n}, l_{n}\right)\right],
$$

where the $l_{i}$ correspond to imaginary times, the $t_{i}$ decreasing $0 \leqslant t_{i} \leqslant \beta$, and the $\psi\left(x_{i}, t_{i}\right)$ are obtained from $\psi(x)$ by progagation under $H$ for an imaginary time $l_{i}$. We now expunge this definition of the $\psi\left(x_{i}, t_{i}\right)$ from our memory and follow an alternate line of development.

We define $\psi(x, l) \equiv \psi(x)$, the $l$ introduced only as a label to enable us to time order. $H(t)$ is $H$ expressed in terms of the $\psi(l)$ and $\psi(l)$ :

$$
\begin{aligned}
& H(l)=(-1 / 2 M) \bar{\psi}(z, l) \nabla^{2} \psi(z, l)+\mu \bar{\psi}(z, l) \psi(z, l) \\
& +\frac{1}{2}: \Psi \psi\left(z_{1}, t\right) V\left(z_{1}, z_{2}\right) \psi \psi\left(z_{2}, t\right): \\
& \operatorname{Tr}\left[T \exp \left[-\int_{0}^{\beta} H(t) d t\right] \bar{\psi}\left(x_{1}, l_{1}\right) \cdots \psi\left(x_{n}, l_{n}\right)\right]
\end{aligned}
$$

with $T$ the time-ordering operation familiar to physicists. In fact (1.4) is essentially the interaction representation with the interaction taken to be the full Hamiltonian-so the interaction fields have no true time dependence, as our $\psi(x, t)$. Taking (1.4) as a serious expression to manipulate and perform estimates with is the heart of the semi-Euclidean approach.

We also want an expression for the path space measure generated by the one-particle free Hamiltonian

$$
\left(\exp \left[-s\left(-1 / 2 M \nabla^{2}+\mu\right)\right] \psi\right)(y)=\int d x \int d \mu_{x, y}^{s} \psi(x),
$$

where $\int d \mu_{x, y}^{s}$ is a measure on paths $x_{p}(l), 0 \leqslant l \leqslant s$, 
connecting $x$ and $y$ (and lying in $V$ ).

\section{EXPANSION STEPS}

In this paper we consider only two operations. The first of these, differentiation of the exponent, assumes $H(t)$ depends on a parameter $\lambda$, and so we write $H_{\lambda}(t)$. This happens by allowing $V\left(z_{1}, z_{2}\right)$ to depend on $\lambda$, and so, $V_{\lambda}\left(z_{1}, z_{2}\right)$. We write $(1.4)$ as

$$
\operatorname{Tr}\left[T \exp \left(-\int_{0}^{\beta} H_{\lambda}(t) d t\right) R\right] .
$$

$R$ a polynomial in fields.

\section{Differentiation of the exponent}

$$
\begin{aligned}
\frac{d}{d \lambda} \operatorname{Tr}\left[T \exp \left(-\int_{0}^{\beta} H_{\lambda}(t) d t\right) R\right] \\
\quad=\int_{0}^{\beta} d s \operatorname{Tr}\left(T \exp \left(-\int_{0}^{\beta} H_{\lambda}(t) d t\right) \frac{d H_{\lambda}(s)}{d \lambda} R\right) .
\end{aligned}
$$

The other operation is a pull-through operation. (The pull-through operation used in Refs. 1 and 2, different from the one presented here, may be useful in some circumstances.)

\section{Pull-through formula}

$$
\begin{aligned}
T \exp \left(-\int_{a}^{b} H(t) d t\right) \bar{\psi}(x, a) \\
=T \int d y \int d \mu_{x, y}^{(b-a)} \bar{\psi}(y, b) \\
\quad \times \exp \left(-\int_{a}^{b}\left[H(l)+V\left(x_{p}(t), z\right) \bar{\psi} \psi(z)\right] d t\right) .
\end{aligned}
$$

We will not write the similar expression for $\psi$ and more general potentials.

This pull-through formula provides the connection between the semi-Euclidean formulation and the work of Ginibre, as we will see in the next section.

To prove (2.3), we consider the equality

$$
\begin{aligned}
T \int d y \int d \mu_{x, y}^{(b\lrcorner a)} \psi(y, b) \exp \left(-\int_{a}^{b}\left[H(t)+V\left(x_{p}, z\right) \psi \psi(z)\right] d t\right) \\
-T \exp \left(-\int_{a}^{b} H(t) d t\right) \psi(x, a) \\
=\int_{a}^{b} d s \frac{d}{d s}\left[T \exp \left(-\int_{s}^{b} H(t) d t\right) \int d y \int d \mu_{x, y}^{(s-a)} \bar{\psi}(y, s)\right. \\
\left.\quad \times \exp \left(-\int_{a}^{s}\left(H(t)+V\left(x_{p}, z\right) \psi \psi\right) d t\right)\right]
\end{aligned}
$$

and verify that the differentiation with respect to $s$ in the brackets gives zero.

\section{GINIBRE-TYPE EXPANSION}

For clarity we confine our attention to the following correlation function $F\left(x_{2}, t_{2}, x_{1}, t_{1}\right)$ :

$$
F=\operatorname{Tr}\left(T \exp \left[-\int_{0}^{\beta} H(t) d t\right] \psi\left(x_{2}, t_{2}\right) \bar{\psi}\left(x_{1}, t_{1}\right)\right)
$$

with $\beta>t_{2}>t_{1}>0$. We now use the pull-through formula (2.3) to move $\bar{\psi}(x, t)$ to the left. We may also use the relation

$$
\bar{\psi}(x, 0)=\bar{\psi}(x, \beta),
$$

following from commutativity of the trace-see $(4.1)$ in

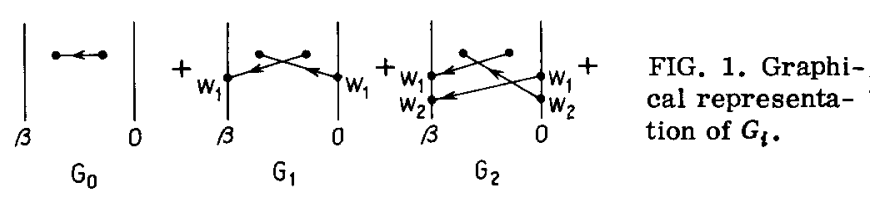

the next section-giving rise to well-known periodicity of our correlation functions. As $\bar{\psi}$ is continuously moved to left, and re-entered at right by (3.2), a series of terms is generated by the possibility of contraction with $\psi$ after any number of sweeps.

$$
\psi(x) \bar{\psi}(y)-\epsilon \bar{\psi}(y) \psi(x)=\delta(x-y)
$$

with $\epsilon=1$ for bosons and $\epsilon=-1$ for fermions. (3.1) then becomes

$$
F=G_{0}+G_{1}+G_{2}+\cdots+G_{N}+R_{N+1} .
$$

The $G_{i}$ are represented graphically in Fig. 1. Expressions for $G_{0}, G_{1}$, and $G_{N}$ follow:

$$
\begin{aligned}
& G_{0}=\operatorname{Tr}\left[T \int d \mu_{x_{1}, x_{2}}^{\left(t_{2}-t_{1}\right)} \exp \left(-\int_{0}^{\beta} H(t) d t\right)\right. \\
& \left.\times \exp \left(-\int_{t_{1}}^{t_{2}}\left[V\left(x_{p}(t), z\right) \bar{\psi} \psi(z)\right] d t\right)\right], \\
& G_{1}=(\epsilon) \operatorname{Tr}\left[T \int d w_{1} \int d \mu_{x_{1}, w_{1}}^{\left(\beta-t_{1}\right)} \int d \mu_{w_{1}, x_{2}}^{t_{2}} \exp \left(-\int_{0}^{\beta} H(t) d t\right)\right. \\
& \times \exp \left(-\int_{0}^{t_{1}} V\left(x_{p_{1}}, z\right) \psi \psi(z) d t\right) \exp \left(-\int_{t_{2}}^{\beta} V\left(x_{p_{0}}, z\right) \bar{\psi} \psi(z) d t\right) \\
& \times \exp \left(-\int_{t_{1}}^{t_{2}}\left[V\left(x_{p_{0}}, z\right) \psi \psi(z)+V\left(x_{p_{1}}, z\right) \bar{\psi} \psi(z)\right.\right. \\
& \left.\left.\left.+V\left(x_{p_{0}}, x_{p_{1}}\right)\right] d t\right)\right] \\
& G_{N}=(\epsilon)^{N} \operatorname{Tr}\left\{T \int d w_{1} \operatorname{\infty o\circ } d w_{N} \int d \mu_{x_{1}, w_{1}}^{\left(\beta-t_{1}\right)} \cdots d \mu_{w_{i}, w_{i+1}}^{\beta}\right. \\
& \cdots d \mu_{w_{N}, x_{2}}^{t_{2}} \exp \left(-\int_{0}^{\beta} H(t) d t\right) \\
& \times \exp \left[-\int_{0}^{t_{1}}\left(\sum_{j=1}^{N} V\left(x_{p_{j}}, z\right) \bar{\psi} \psi(z)+\sum_{1 \leqslant i<j}^{N} V\left(x_{p_{i}}, x_{p_{j}}\right)\right) d t\right] \\
& \times \exp \left[-\int_{t_{2}}^{\beta}\left(\sum_{j=0}^{N-1} V\left(x_{p_{j}}, z\right) \bar{\psi} \psi(z)\right.\right. \\
& \left.\left.+\sum_{0 \leqslant i<j}^{N-1} V\left(x_{p_{i}}, x_{p_{j}}\right)\right) d t\right] \\
& \times \exp \left[-\int_{t_{1}}^{t_{2}}\left(\sum_{j=0}^{N} V\left(x_{p_{j}}, z\right) \bar{\psi} \psi(z)+\sum_{0 \leqslant i<j}^{N} V\left(x_{p_{i}}, x_{p_{j}}\right) d t\right]\right\} .
\end{aligned}
$$

This expansion may be compared to the expansions of Ginibre in Ref. 5. We will not here make explicit an expression for $R_{N}$. However, in Appendix $\mathrm{C}$ an explicit expression for $R_{1}$ is given, and estimated as an example of the operator estimates given in the next section. In Appendix B $G_{0}$ is similarly estimated. Future applications of the semi-Euclidean formalism depend on our ability to control estimates-- Appendix B and Appendix $C$ are simpler than the estimates needed in the cluster expansion, but use the same basic techniques. 


\section{OPERATOR ESTIMATES}

We collect here, first, well-known estimates we will use concerning traces; recall an operator estimate from Ref. 1; and finally present a cute new estimate for traces in a theorem.

We begin with the well-known facts:

$$
\begin{aligned}
& \operatorname{Tr}(A B)=\operatorname{Tr}(B A), \\
& \left\{\begin{array}{l}
A \geqslant B \Rightarrow \operatorname{Tr}\left(e^{-A}\right) \leqslant \operatorname{Tr}\left(e^{-B}\right), \\
A \geqslant 0 \Rightarrow \operatorname{Tr}(A) \geqslant 0, \\
A \geqslant B \Rightarrow \operatorname{Tr}\left(C A C^{*}\right) \geqslant \operatorname{Tr}\left(C B C^{*}\right),
\end{array}\right. \\
& |\operatorname{Tr}(A C)| \leqslant\left[\operatorname{Tr}\left(A A^{*}\right)\right]^{1 / 2} \cdot\left[\operatorname{Tr}\left(C C^{*}\right)\right]^{1 / 2}, \\
& |\operatorname{Tr}(A B C)| \leqslant\left[\operatorname{Tr}\left(A A^{*}\right)\right]^{1 / 2} \cdot\|B\| \cdot\left[\operatorname{Tr}\left(C C^{*}\right)\right]^{1 / 2} \text {. }
\end{aligned}
$$

\section{We recall from Ref. 1:}

Estimate: Let $H(t) \geqslant C(t)$ with $C(t)$ a numerical function. Then

$$
\left\|T \exp \left(-\int_{0}^{\beta} H(t) d t\right)\right\| \leqslant \exp \left(-\int_{0}^{\beta} C(t) d t\right) .
$$

The following theorem is an estimate similar to this last estimate, but for traces.

Theorem: Let $H(t) \geqslant \bar{H}+C(t)$ with $C(t)$ a numerical function and $\bar{H}$ a $t$-independent operator. Then

$\left|\operatorname{Tr}\left(T \exp \left[-\int_{0}^{\beta} H(t) d t\right]\right)\right| \leqslant \operatorname{Tr}\left(\exp (-\beta \bar{H}) \cdot \exp \left(-\int_{0}^{\beta} C(t) d t\right)\right.$.

A proof of this theorem is presented in Appendix A.

\section{APPENDIX A: PROOF OF TIME-ORDERED TRACE INEQUALITY}

The inequality follows by taking limits in a discrete form of the result, stated in the following lemma, and using (4.2). The lemma is a special case of Corollary 3.2 in Ref. 8.

Lemma:

$$
\left|\operatorname{Tr}\left(A_{1} \cdots A_{2^{N}}\right)\right| \leqslant \prod_{i}\left\{\operatorname{Tr}\left[\left(A_{i} A_{i}^{*}\right)^{2^{N-1}}\right]\right\}^{1 / 2^{N}} .
$$

(The restriction of the product of $A_{i}$ 's to contain a power of two elements is a function of our method of proof.)

Proof: We prove the result by induction on $N$. First for $N=1$ :

$$
\left|\operatorname{Tr}\left(A_{1} A_{2}\right)\right| \leqslant\left[\operatorname{Tr}\left(A_{1} A_{1}^{*}\right)\right]^{1 / 2} \circ\left[\operatorname{Tr}\left(A_{2} A_{2}^{*}\right)\right]^{1 / 2} .
$$

This is just $(4,5)$. Assume (A1) holds for $N$ and proceed to the $N+1$ case:

$$
\left|\operatorname{Tr}\left(A_{1} \cdots A_{2^{N+1}}\right)\right|=\left|\operatorname{Tr}\left(B_{1} \cdots B_{2^{N}}\right)\right|
$$

with

$$
\begin{aligned}
B_{i}= & A_{2 i-1} A_{2 i} \\
& \leqslant \prod_{i}\left\{\operatorname{Tr}\left[\left(B_{i} B_{i}^{*}\right)^{2^{N-1}}\right]\right\}^{1 / 2^{N}}
\end{aligned}
$$

by the induction hypothesis. We consider one of these terms, $i=1$, for notational simplicity:

$$
\operatorname{Tr}\left[\left(B_{1} B_{1}^{*}\right)^{2^{N-1}}\right]=\operatorname{Tr}\left[\left(A_{1}^{*} A_{1} A_{2} A_{2}^{*}\right)^{2^{N-1}}\right] .
$$

(4.1) was here used. By the induction hypothesis,

$$
\leqslant\left\{\operatorname{Tr}\left[\left(A_{2} A_{2}^{*}\right)^{2^{N}}\right]\right\}^{1 / 2}\left\{\operatorname{Tr}\left[\left(A_{1}^{*} A_{1}\right)^{2^{N}}\right]\right\}^{1 / 2} \text {. }
$$

Substituting (A6) back in (A4) we obtain the $(N+1)$ th relation, completing the induction.

\section{APPENDIX B: ESTIMATE FOR $G_{0}$}

$G_{0}$ is given in (3.5). To obtain an estimate for $G_{0}$, we require two estimates; we write as follows:

$$
\begin{aligned}
H_{0} / 2 & +\frac{1}{2} \int d z_{1} d z_{2}: \bar{\psi} \psi\left(z_{1}\right) V\left(z_{1}, z_{2}\right) \bar{\psi} \psi\left(z_{2}\right): \geq K_{0}, \\
H_{0} / 2 & +\frac{1}{2} \int d z_{1} d z_{2}: \bar{\psi} \psi\left(z_{1}\right) V\left(z_{1}, z_{2}\right) \bar{\psi} \psi\left(z_{2}\right): \\
& +\int d z V(x, z) \bar{\psi} \psi(z) \geqslant K_{1} .
\end{aligned}
$$

We do not discuss evaluation of $K_{0}$ and $K_{1}$ here, as depending on $V$, the volume, and the interaction. However, it is important to note that in these evaluations the kinetic energy arising in the Fermion case may be exploited (see Ref, 3). We now use (4.8) to obtain

$$
\begin{aligned}
\left|G_{0}\right| & \leqslant \operatorname{Tr}\left[\exp \left(-\beta H_{0} / 2\right)\right] \cdot \exp \left[-K_{1}\left(t_{2}-t_{1}\right)\right] \\
& \times \exp \left[-K_{0}\left(\beta-\left(t_{2}-t_{1}\right)\right)\right] \\
& \cdot \frac{(M / 2 \pi)^{3 / 2}}{\left|t_{2}-t_{1}\right|^{3 / 2}} \exp \left(-\frac{M}{2} \frac{\left|x_{2}-x_{1}\right|^{2}}{\left(t_{2}-t_{1}\right)}\right) .
\end{aligned}
$$

\section{APPENDIX C: Estimate for $R_{1}$}

We graphically represent $R_{1}$ in Fig. 2. For definiteness we require $2 / 3 \beta>t_{2}>t_{1}>\beta / 3$, this is not essential. Having pulled-through $\bar{\psi}$ past $t_{2}$ without contraction with $\psi$, we stop at $2 / 3 \beta$ and then pull-through $\psi$ to $1 / 3 \beta$. The resulting expression for $R_{1}$ is

$$
\begin{aligned}
R_{1}= & \epsilon \int d w_{1} d w_{2} \int d \mu_{x_{1}, w_{1}}^{\left(2 / 3 \beta-t_{1}\right)} \int d \mu_{w_{2}, x_{2}}^{\left(t_{2}-1 / 3 \beta\right)} \\
& \times \operatorname{Tr}\left[T \exp \left(-\int_{2 / 3 \beta}^{\beta} H(t) d t\right) \bar{\psi}\left(w_{1}, 2 / 3 \beta\right)\right. \\
& \cdot \exp \left(-\int_{t_{2}}^{2 / 3 \beta}\left[H(t)+V\left(x_{p_{1}}, z\right) \bar{\psi} \psi(z)\right] d t\right) \\
& \cdot \exp \left(-\int_{t_{1}}^{t_{2}}\left[H(t)+V\left(x_{p_{1}}, z\right) \bar{\psi} \psi(z)\right.\right. \\
& \left.\left.+V\left(x_{p_{2}}, z\right) \bar{\psi} \psi(z)+V\left(x_{p_{1}}, x_{p_{2}}\right)\right] d t\right) \\
& \cdot \exp \left(-\int_{\beta / 3}^{t_{1}}\left[H(t)+V\left(x_{p_{2}}, z\right) \bar{\psi} \psi(z)\right] d t\right) \\
& \left.\times \psi\left(w_{2}, 1 / 3 \beta\right) \exp \left(-\int_{0}^{\beta / 3} H(t) d t\right)\right] .
\end{aligned}
$$

We abbreviate this as

$$
\begin{aligned}
R_{1}= & \epsilon \int d w_{1} d w_{2} \int d \mu_{1} d \mu_{2} \exp \left(-\int_{t_{1}}^{t_{2}} V\left(x_{p_{1}}, x_{p_{2}}\right) d t\right) \\
& \times \operatorname{Tr}\left[T E_{1} \bar{\psi}\left(w_{1}\right) E_{2} E_{3} E_{4} \psi\left(w_{2}\right) E_{5}\right],
\end{aligned}
$$

where the $E_{i}$ are the obvious exponentials, except that, as indicated, a portion of $E_{3}$ has been separated out explicitly. We use (4.6):

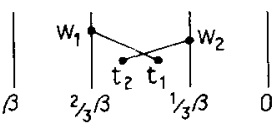

FIG. 2. Graphical representation of $R_{1}$. 


$$
\begin{aligned}
\left|R_{1}\right| & \leqslant \int d w_{1} d w_{2} \int d \mu_{1} d \mu_{2} \exp \left(-\int_{t_{1}}^{t_{2}} V(1,2) d t\right) \\
& \times\left\{\operatorname{Tr}\left[\left(T E_{1}\right) \bar{\psi}\left(w_{1}\right) \psi\left(w_{1}\right)\left(T^{*} E_{1}^{*}\right)\right]\right\}^{1 / 2}\left\|E_{2} E_{3} E_{4}\right\| \\
& \times\left\{\operatorname{Tr}\left[\left(T^{*} E_{5}^{*}\right) \bar{\psi}\left(w_{2}\right) \psi\left(w_{2}\right)\left(T E_{5}\right)\right]\right\}^{1 / 2}
\end{aligned}
$$

using

$$
2 x y \leqslant x^{2}+y^{2}
$$

we get

$$
2\left|R_{1}\right| \leqslant A+B
$$

with

$$
\begin{aligned}
A= & \int d w_{1} \int d w_{2} \int d \mu_{1} \int d \mu_{2} \exp \left(-\int_{t_{1}}^{t_{2}} V(1,2) d t\right) \\
& \times \operatorname{Tr}\left[\left(T E_{1}\right) \bar{\psi}\left(w_{1}\right) \psi\left(w_{1}\right)\left(T^{*} E_{1}^{*}\right)\right]\left\|E_{2} E_{3} E_{4}\right\|
\end{aligned}
$$

and a similar expression for $B$.

Let

$$
\left|\int d \mu_{1} \int d \mu_{2} \exp \left(-\int_{t_{1}}^{t_{2}} V(1,2) d t\right)\right| \leqslant C
$$

for all $w_{1}$ and $w_{2}$. This inequality, a statement concerning two particles interacting by a mutual potential, can be estimated by standard methods. We get

$$
\begin{aligned}
A & \leqslant C \vee \exp \left\{-\left[\left(2 / 3 \beta-t_{2}\right) K_{1}+\left(t_{2}-t_{1}\right) K_{2}+\left(t_{1}-\beta / 3\right) K_{1}\right]\right\} \\
& \times \operatorname{Tr}\left[\exp \left(-2 / 3 \beta H_{0}\right) N\right] .
\end{aligned}
$$

$K_{2}$ is defined similarly to $K_{0}$ and $K_{1}$. The methods of Appendices $B$ and $C$ can be applied in much more complicated situations, and can, very essentially, include localization.

*This work was supported in part by NSF Grant GP-17523. ${ }^{1}$ D. Brydges and P. Federbush, J. Math. Phys. 15, 730 (1974).

${ }^{2}$ D. Brydges, J. Math. Phys, 16, 1649 (1975).

${ }^{3} \mathbf{P}$. Federbush, A new approach to the stability of matter Problem. I and II, J. Math. Phys. 16, 347, 706 (1975). ${ }^{4}$ F. J. Dyson and A. Lenard, J. Math. Phys. 8, 423 (1967); 9,698 (1968).

5 J. Ginibre, "Some Applications of Functional Integration in Statistical Mechanics," in Statistical Mechanics and Quantum Field Theory, Les Houches 1970, edited by C. DeWitt and R. Stora (Gordon and Breach, New York, 1971).

${ }^{6}$ F. J. Dyson, J. Math. Phys. 8, 1538 (1967).

${ }^{7} \mathrm{~J}$. Glimm, A. Jaffe, and T. Spencer, "The Cluster Expansion, in: Constructive Quantum Field Theory," in The 1973

"Ettore Majorana" International School of Mathematical Physics, edited by G. Velo and A. Wightman (SpringerVerlag, Berlin, 1973).

${ }^{8}$ R. A. Kunze, Trans. Amer. Math. Soc. 89, 519 (1958). 